\title{
A successfully treated case of primary purulent pericarditis complicated by cardiac tamponade and pneumopericardium
}

\author{
Jong Wook Beom'1, Yeekyoung Ko', Ki Yung Boo', Jae-Geun Lee', Joon Hyouk Choi', Seung-Jae Joo', \\ Ji Hwan Moon'2, Su Wan Kim ${ }^{3}$, Song-Yi Kim ${ }^{1}$ \\ ${ }^{1}$ Division of Cardiology, Department of Internal Medicine, ${ }^{2}$ Department of Internal Medicine, and ${ }^{3}$ Department of Thoracic and Cardiovascular Surgery, Jeju \\ National University Hospital, Jeju National University School of Medicine, Jeju, Korea
}

Acute pericarditis is caused by various factors, but purulent pericarditis is rare. Primary purulent pericarditis in immunocompetent hosts is very rare in the modern antibiotics era. We report a successfully treated case of primary purulent pericarditis complicated with cardiac tamponade and pneumopericardium in an immunocompetent host. A 69-year-old female was referred from another hospital because of pleuritic chest pain with a large amount of pericardial effusion. She was diagnosed with acute pericarditis accompanied by cardiac tamponade. We performed emergency pericardiocentesis, with drainage of $360 \mathrm{ml}$ of bloody pericardial fluid. The culture grew Streptococcus anginosus, confirming the diagnosis of acute purulent pericarditis. We performed pericardiostomy because cardiomegaly and pneumopericardium were aggravated after removal of the pericardial drainage catheter. The patient received antibiotics for a total of 23 days intravenously and was discharged with oral antibiotic therapy. Purulent pericarditis is one of the rare forms of pericarditis and is lifethreatening. A multimodality approach is required for proper diagnosis and treatment of this disease.

Key Words: Cardiac tamponade; Pericardiocentesis; Pericarditis; Streptococcus anginosus

Acute pericarditis may be caused by infectious, noninfectious, or autoimmune disorders. Though purulent pericarditis is rare, it is a rapidly progressive infection with a mortality rate of $20 \%$ to $30 \%$ [1]. In the modern antibiotics era, purulent pericarditis is usually associated with nosocomial bloodstream infections, thoracic surgery, immunosuppression, or underlying pericardial disease [2,3]. In immunocompetent hosts, primary purulent pericarditis without evidence of underlying infection elsewhere and recent trauma history is very rare. Until now, cardiac tamponade and pneumopericardium have not been reported in patients with purulent pericarditis. We present a case of a 69-year-old immunocompetent previously healthy adult patient who was diagnosed as having primary acute purulent pericarditis complicated by cardiac tamponade and pneumopericardium.

\section{Case Report}

Received: May 9, 2020

Revised: July 2, 2020

Accepted: July 7, 2020

Corresponding author Song-Yi Kim

Division of Cardiology, Department of Internal Medicine, Jeju National University School of Medicine, 15 Aran 13-gil, Jeju 63241, Korea Tel: +82-64-717-1296

Fax: +82-64-717-1131

E-mail: ttoromom@jejunu.ac.kr

Copyright () 2021 The Korean Society of Critical Care Medicine

This is an Open Access article distributed under the terms of Creative Attributions Non-Commercial License (https:// creativecommons.org/li-censes/by-nc/4.0/) which permits unrestricted noncommercial use, distribution, and reproduction in any medium, provided the original work is properly cited. 


\section{CASE REPORT}

This case report was approved by Institutional Review Board of Jeju National University Hospital (IRB No. JEJUNUH 201912-010). We obtained written informed consent from the patient.

A 69-year-old female was referred from another hospital because of a large amount of pericardial effusion. She had complained of pleuritic chest pain of 4 days' duration. The pain started from the back, moved to the substernal area, and was worse when breathing or moving. In the emergency department, the patient complained of chest pain and difficulty breathing but denied fever, chills, toothache, cough, hemoptysis, and urinary symptoms. She also denied any history of recent dental caries, respiratory infections, chest wall trauma, surgeries, travel, or exposure to sick contacts. She was on medication for hypertension and diabetes mellitus and had been using an inhaled corticosteroid for asthma for 2 months. She denied any alcohol, smoking, or illicit drug use.

At the time of presentation, the patient was well-oriented and had a blood pressure of $98 / 70 \mathrm{~mm} \mathrm{Hg}$, a heart rate of 54 beats/min, a respiratory rate of $24 /$ minute, and a body temperature of $37.2^{\circ} \mathrm{C}$. However, 30 minutes after initial presentation, her blood pressure decreased to $79 / 58 \mathrm{~mm} \mathrm{Hg}$, and her heart rate increased to 100 beats/min. Cardiopulmonary examination showed decreased heart sounds and a coarse breathing sound over the bilateral lower lung field. Jugular venous distension and hepato-jugular reflux were observed. Blood test results were as follows: white blood cell (WBC) $20.4 \times 103 / \mu \mathrm{l}$, hemoglobin $12.3 \mathrm{~g} / \mathrm{dl}$, hematocrit $36.5 \%$, platelets $239 \times 10^{3} / \mu \mathrm{l}$, C-reactive protein $13.83 \mathrm{mg} / \mathrm{dl}$, procalcitonin $5.14 \mathrm{mg} / \mathrm{ml}$, blood urea nitrogen $50.9 \mathrm{mg} / \mathrm{dl}$, serum creatinine $1.92 \mathrm{mg} / \mathrm{dl}$, pro-brain natriuretic peptide $1,833 \mathrm{pg} / \mathrm{ml}$, cre- atine kinase-MB $3.27 \mathrm{ng} / \mathrm{ml}$, hs-troponin T $0.088 \mathrm{ng} / \mathrm{ml}$, aspartate aminotransferase $221 \mathrm{IU} / \mathrm{L}$, alanine aminotransferase 193 IU/L, hemoglobin Alc 7.2\%, and negative HIV antigen/ antibody test. Chest radiography revealed cardiomegaly with $70 \%$ cardiothoracic ratio and bilateral pleural effusion (Figure 1A). An electrocardiogram showed sinus tachycardia, lowvoltage QRS complexes, and diffuse ST-segment elevations (Figure 1B). Chest computed tomography (CT) revealed a moderate amount of pericardial effusion with diffuse pericardial enhancement that compressed the right ventricle (Figure $2 \mathrm{~A})$. In the parasternal long axis view of echocardiography, a large amount of pericardial effusion with $2 \mathrm{~cm}$ compression of the right ventricle was observed (Figure 2B). The patient was diagnosed with acute pericarditis accompanied by cardiac tamponade. An emergency pericardiocentesis was performed, and approximately $360 \mathrm{ml}$ of bloody pericardial effusion was drained. After pericardiocentesis, the amount of pericardial effusion decreased by $1.5 \mathrm{~cm}$ in the parasternal long axis view of echocardiography (Figure 2C). Tests for culture, cell analysis, cytology, and tuberculosis were performed with the pericardial fluid. Laboratory findings revealed acute purulent pericarditis with $200,000 / \mu \mathrm{l}$ of WBC composed of $99 \%$ segmented neutrophils. No bacteria were found via a gram stain of the pericardial fluid. However, third-generation cephalosporin and ibuprofen were empirically used. On the patient's fourth in-hospital day, Streptococcus anginosus was identified in blood and pericardial fluid cultures, and the infection was susceptible to ceftriaxone and levofloxacin but resistant to ampicillin.

On the 4th in-hospital day, pericardial effusion was drained from the pericardial catheter, and the catheter was removed. Unfortunately, chest radiography comparisons on the 5th and 7th in-hospital days demonstrated that severe cardiomegaly,
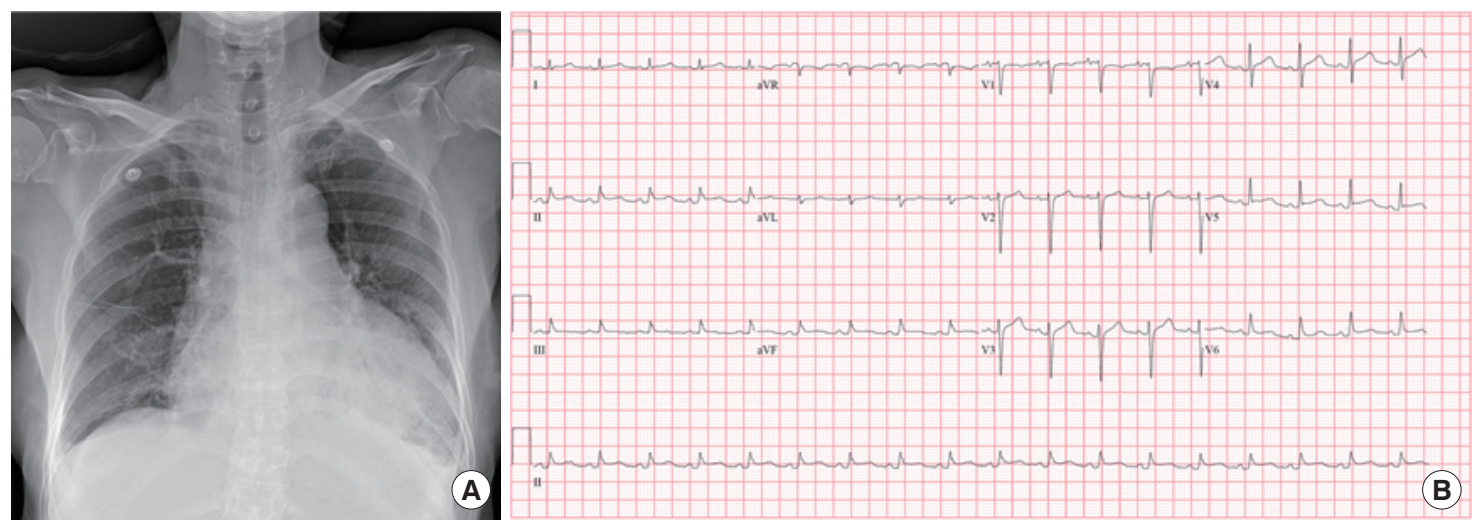

Figure 1. Baseline examination. (A) Chest radiography showing marked cardiomegaly with $70 \%$ cardiothoracic ratio and bilateral pleural effusion. (B) Electrocardiogram showing low-voltage QRS and diffuse ST elevation. 

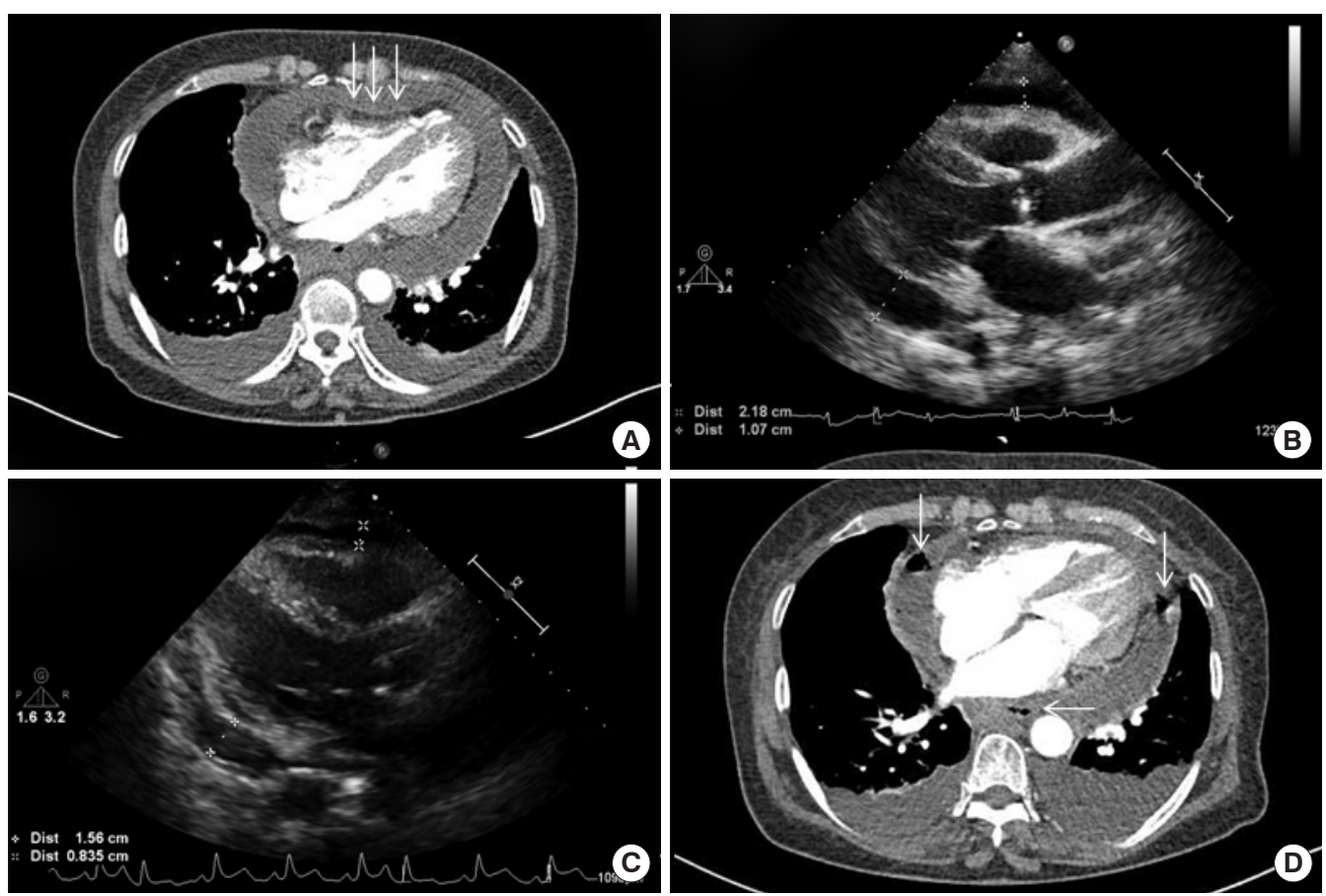

Figure 2. Changes in chest computed tomography (Chest CT) and echocardiogram. (A) Chest CT scan showing compression of the right ventricle (white arrows) due to pericardial effusion. (B) Transthoracic echocardiogram showing massive pericardial effusion. (C) After pericardiocentesis, transthoracic echocardiogram showing a decreased amount of pericardial effusion. (D) Chest CT scan showing loculated pericardial effusion with multi-focal air bubbles (white arrows) in the pericardial space.
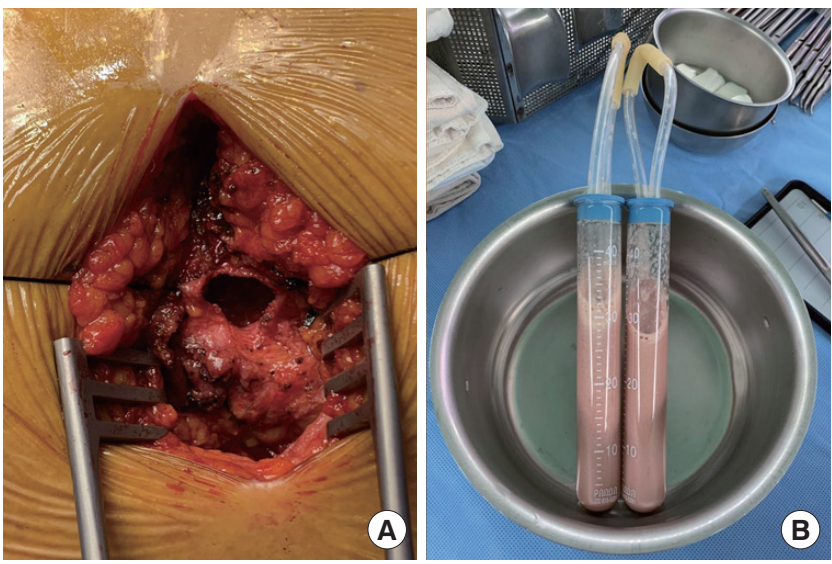

Figure 3. Surgical finding. (A) Pericardiostomy through the subxiphoid approach. (B) Pericardial effusion with grayish pus.

and new air bubbles appeared surrounding the main pulmonary artery. Chest CT showed loculated and increasing pericardial effusion with multi-focal air bubbles that indicated aggravated purulent pericarditis (Figure 2D). It seemed that the patient's clinical condition worsened due to abscess formation despite the use of appropriate antibiotics.

Pericardiostomy was performed through the subxiphoid approach on the 9th in-hospital day. There were loose adhesions surrounding the whole heart, especially on the cardiac base and right atrium. After careful adhesiolysis, approximately $200 \mathrm{ml}$ of grayish pus was drained (Figure 3). Massive saline irrigation was performed, a curved chest tube was inserted below the left ventricular apex, and a Jackson-Pratt catheter was inserted beside the right atrium.

The patient's condition gradually improved, and the drains were removed two weeks after the operation. The patient was discharged 24 days after admission with oral antibiotics for 14 days. The total duration of antibiotic therapy was 36 days, and the patient is doing well without any evidence of recurrence.

\section{DISCUSSION}

We report a case of primary purulent pericarditis complicated by cardiac tamponade and pneumopericardium and caused by $S$. anginosus in an immunocompetent, previously healthy adult female patient. Although the patient was using an inhaled corticosteroid, it is known to have few systemic side effects and has not been reported to increase infections other than pneumonia [4]. The patient had been complaining of pleuritic chest pain that was gradually worsening for 4 days. She was diagnosed with cardiac tamponade associated with a large amount of pericardial effusion and underwent emergency pericardiocentesis. Despite proper treatment, chest ra- 
diography showed worsening of cardiomegaly and pneumopericardium, so a pericardiostomy was performed.

Purulent pericarditis, a localized infection within the pericardial space, is a rare disease that is associated with lifethreatening complications such as cardiac tamponade [5]. In the pre-antibiotic era (1889-1943), it occurred mainly in young adult patients with underlying infectious disease. But in the modern-antibiotic era (1944-1975), it is most common in elderly adult patients with preexisting pericardial diseases such as uremia or tumor [2]. Other predisposing factors include immunosuppression, alcohol abuse, thoracic surgery, and chest trauma [6].

Common pathogens of purulent pericarditis were Staphylococcus aureus (36\%) and Streptococcus pneumonia (21\%) [5]. Before the advent of penicillin, pneumococcus was the most common pathogen in the setting of direct spread from an intrathoracic focus [7]. However, after penicillin became available, the number of pneumococcal infections has decreased, including that of purulent pericarditis [8]. Gram-negative, anaerobic organisms and fungal pathogens are increasing in proportion as causes of primary purulent pericarditis. S. anginosus is a subgroup of viridans streptococci that is part of the normal flora of the mouth, sinuses, throat, feces, and vagina [9]. However, it can cause a number of infections and has a tendency toward abscess formation. It is also often seen as part of a polymicrobial infection in patients with oral, head and neck, and abdominal infections. There are several ways by which this strain gains access to the thoracic space: aspiration of oral secretions, direct implantation during surgery or trauma, by extension from a contiguous focus such as a subphrenic abscess, and via the bloodstream from a distant focus [10]. Our case involved primary purulent pericarditis in an immunocompetent adult who was previously healthy, caused by S. anginosus. The origin of S. anginosus, the pathogen of infection, was uncertain, and pericarditis occurred spontaneously without evidence of any other infection.

This case highlights the importance of physical examination and early diagnostic testing such as echocardiography and chest CT. This allows a faster diagnosis, performance of pericardiocentesis, and administration of antibiotics. When the patient's condition worsened even with proper treatment, chest CT was promptly examined and pericardiostomy immediately performed. If not treated in time, purulent pericarditis can have a mortality rate approaching $100 \%$ [11].

In conclusion, we report an extremely rare case of primary purulent pericarditis complicated by cardiac tamponade and pneumopericardium caused by S. anginosus. We emphasize the importance of clinical suspicion, early diagnostic testing, and multimodality therapy (pericardiocentesis, pericardiostomy, and appropriate antibiotic therapy) to ensure patient survival.

\section{CONFLICT OF INTEREST}

No potential conflict of interest relevant to this article was reported.

\section{ORCID}

Jong Wook Beom https://orcid.org/0000-0002-4503-6133

Yeekyoung Ko https://orcid.org/0000-0002-2496-7115

Ki Yung Boo https://orcid.org/0000-0002-8098-9132

Jae-Geun Lee https://orcid.org/0000-0003-4915-9584

Joon Hyouk Choi https://orcid.org/0000-0003-4245-1582

Seung-Jae Joo https://orcid.org/0000-0002-4322-0703

Ji Hwan Moon https://orcid.org/0000-0001-8106-0029

Su Wan Kim https://orcid.org/0000-0002-6531-7266

Song-Yi Kim https://orcid.org/0000-0002-1170-8165

\section{AUTHOR CONTRIBUTIONS}

Conceptualization: JWB, SYK, SWK. Data curation: JWB, SYK. Methodology: JWB, SWK. Project administration: SYK, SJJ. Writing-original draft: JWB. Writing-review\&editing: all authors.

\section{REFERENCES}

1. Augustin P, Desmard M, Mordant P, Lasocki S, Maury JM, Heming N, et al. Clinical review: intrapericardial fibrinolysis in management of purulent pericarditis. Crit Care 2011;15: 220.

2. Klacsmann PG, Bulkley BH, Hutchins GM. The changed spectrum of purulent pericarditis: an 86 year autopsy experience in 200 patients. Am J Med 1977;63:666-73.

3. Hall IP. Purulent pericarditis. Postgrad Med J 1989;65:444-8.

4. Drummond MB, Dasenbrook EC, Pitz MW, Murphy DJ, Fan E. Inhaled corticosteroids in patients with stable chronic obstructive pulmonary disease: a systematic review and metaanalysis. JAMA 2008;300:2407-16.

5. Parikh SV, Memon N, Echols M, Shah J, McGuire DK, Keeley EC. Purulent pericarditis: report of 2 cases and review of the literature. Medicine (Baltimore) 2009;88:52-65.

6. Skiest DJ, Steiner D, Werner M, Garner JG. Anaerobic pericar- 
ditis: case report and review. Clin Infect Dis 1994;19:435-40.

7. Go C, Asnis DS, Saltzman H. Pneumococcal pericarditis since 1980. Clin Infect Dis 1998;27:1338-40.

8. Kauffman CA, Watanakunakorn C, Phair JP. Purulent pneumococcal pericarditis: a continuing problem in the antibiotic era. Am J Med 1973;54:743-50.
9. Ruoff KL. Streptococcus anginosus ("Streptococcus milleri"): the unrecognized pathogen. Clin Microbiol Rev 1988;1:102-8.

10. Hocken DB, Dussek JE. Streptococcus milleri as a cause of pleural empyema. Thorax 1985;40:626-8.

11. Ott CL, Hodge S. Gas-forming purulent pericardial effusion. Can J Cardiol 2009;25:e337. 